\title{
Sharp emission from single InAs quantum dots grown on vicinal GaAs surfaces
}

\author{
U. Perinetti, ${ }^{1, a)}$ N. Akopian, ${ }^{1}$ Yu. B. Samsonenko, ${ }^{2,3,4}$ A. D. Bouravleuv, ${ }^{2,3}$ G. E. Cirlin, ${ }^{2,3,4}$ \\ and V. Zwiller ${ }^{1}$ \\ ${ }^{1}$ Quantum Transport, Kavli Institute of Nanoscience, Delft University of Technology, 2628CJ Delft, \\ The Netherlands \\ ${ }^{2}$ A. F. Ioffe Physico-Technical Institute, 194021 St. Petersburg, Russia \\ ${ }^{3}$ St. Petersburg Physics and Technology Center for Research and Education, 194021 St. Petersburg, Russia \\ ${ }^{4}$ Institute for Analytical Instrumentation, 190103 St. Petersburg, Russia
}

(Received 20 February 2009; accepted 6 April 2009; published online 24 April 2009)

\begin{abstract}
We report on optical studies of single InAs quantum dots grown on vicinal GaAs(001) surfaces. To ensure low quantum dot density and appropriate size, we deposit InAs layers 1.4 or 1.5 ML thick, thinner than the critical thickness for Stranski-Krastanov quantum dot formation. These dots show sharp and bright photoluminescence. Lifetime measurements reveal an exciton lifetime of 500 ps. Polarization measurements show an exciton fine structure splitting of $15 \mu \mathrm{eV}$ and allow to identify the exciton and charged exciton transitions with linewidth as narrow as $23 \mu \mathrm{eV}$. () 2009 American Institute of Physics. [DOI: 10.1063/1.3125430]
\end{abstract}

Self-assembled quantum dots (QDs) proved an excellent system for the implementation of quantum bits ${ }^{1-3}$ and as sources of single ${ }^{4,5}$ and entangled photons. ${ }^{6-8}$ The most common technique for growing self-assembled QDs, the Stranski-Krastanov (SK) method, allows for the fabrication of QDs with good optical properties; nearly lifetime limited linewidth of the optical transitions in these dots makes them suitable for single spin measurement ${ }^{9}$ and two photon interference. ${ }^{10}$ The ability of controlling the position of selfassembled QDs would allow for more complex devices, and different attempts ${ }^{11,12}$ have been made in this direction. In particular a growth technique similar to the SK method has been used to produce rows of QDs (Ref. 13) on vicinal GaAs substrates. Although this technique gives some control on the lateral alignment of QDs, the study of the optical properties of such vicinal QDs has been limited to ensemble measurements so far. ${ }^{14,15}$

In this letter, we present a study of the optical properties of single InGaAs QDs, grown on a vicinal GaAs substrate by molecular beam epitaxy (MBE). The low QD density required for addressing single dots has been achieved by selecting a substrate with suitable misorientation angle and by exploring the subcritical regime of InAs island formation. ${ }^{16}$ The high resolution spectroscopic measurements presented in this letter, combined with polarization selective and time resolved measurements, give a first contribution in identifying optical transitions in single QDs of this kind.

In order to grow samples with different QD sizes and densities, four substrates were mounted in the MBE reactor, namely an exactly oriented $\mathrm{GaAs}(001)$ substrate and three GaAs(001) vicinal substrates misoriented by $3^{\circ}, 5^{\circ}$, and $7^{\circ}$ toward the [100] direction. After oxide layer desorption, a GaAs buffer layer was grown at $600{ }^{\circ} \mathrm{C}$ to ensure the translation of multiatomic steps according to the substrate vicinality. A 10 period GaAs/AlGaAs superlattice was then grown to block carrier diffusion to and from the substrate. After growing a $100 \mathrm{~nm}$ thick GaAs layer, the substrate tempera-

${ }^{a)}$ Electronic mail: u.perinetti@tudelft.nl. ture was lowered to $485{ }^{\circ} \mathrm{C}$ and 1.4 or 1.5 InAs monolayers were deposited at a rate of $0.05 \mathrm{ML} / \mathrm{s}$. The growth was then interrupted for $20 \mathrm{~s}$. During this time QDs formed in the subcritical regime, as the InAs layer thickness was below the 1.7 ML critical thickness for SK dot formation. Under the thermodynamically controlled growth conditions typical of this regime, the predicted density is much smaller than the density of commonly used SK dots. ${ }^{17}$ Additionally, the use of vicinal surfaces can allow for lateral ordering of QDs. ${ }^{18,19}$ The structure was finalized by a GaAs $70 \mathrm{~nm}$ cap layer.

We performed photoluminescence (PL) spectroscopy measurements on these eight samples under continuous wave and pulsed laser excitation. The sample grown on the $7^{\circ}$ misoriented substrate with a 1.4 ML InAs layer showed the narrowest emission linewidth and a low QD density, allowing to study single dots. In the following we discuss measurements on this sample only.

In order to measure on a single QD we looked for low density regions of the sample, based on PL spectroscopy. The resolution of our spectrometer is $30 \mu \mathrm{eV}$, considerably smaller than the inhomogeneous distribution in the emission energy of different dots due to size fluctuations. We can therefore resolve a single dot by filtering the emission both spatially and in energy.

A typical spectrum from a QD grown on a $7^{\circ}$ misoriented substrate is shown in Fig. 1, where different optical transitions can be resolved. The line at the highest energy is particularly bright compared to conventional SK dots (without a microcavity) ${ }^{20}$ indicating that the effect of nonradiative processes is relatively small in these structures. Furthermore, the width of this line is smaller than the spectrometer resolution, suggesting these QDs could be used for sophisticated experiments where the selectivity in energy is a crucial requirement, such as spin entanglement in separated quantum systems. $^{21}$

The time evolution of the PL spectrum after pulsed laser excitation [Figs. 1(b) and 1(c)] reveals a lifetime of the different states ranging from 300 to $600 \mathrm{ps}$. The maximum intensity of different transitions occurs at different times sug- 


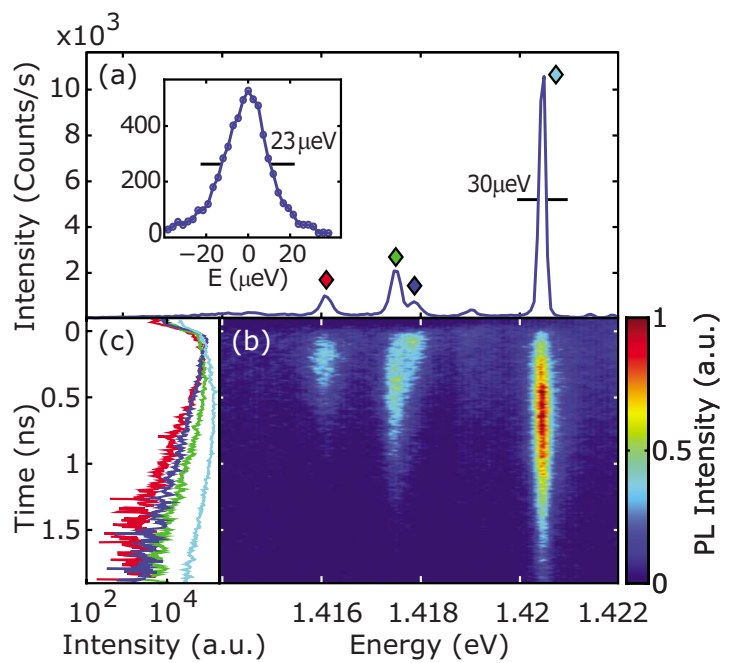

FIG. 1. (Color online) (a) PL spectrum of a single vicinal QD under pulsed laser excitation at $1.647 \mathrm{eV}$. The width of the brightest line is $30 \mu \mathrm{eV}$, limited by the spectrometer resolution. (b) Time resolved PL measurement: a picosecond pulsed laser with a repetition rate of $76 \mathrm{MHz}$ populates the QD via nonresonant excitation. (c) Cuts of the graph in (b) at the wavelengths indicated by the colored diamonds. The inset shows a Fabry-Pérot interferometer measurement on a narrow line from another QD.

gesting that the peaks could correspond to stages of a cascaded emission. Combining measurements of this kind with polarization measurements allows us to identify some of the lines.

In order to identify the exciton (X) and biexciton (XX) transitions in a QD we observe the dependence of the PL spectrum on excitation power. We expect the intensity ratio between the XX and $\mathrm{X}$ transition to increase with excitation power. Spectra taken at different powers [Fig. 2(a)] suggest that the middle line (B) could correspond to the XX transition. However, the time resolved measurement in Fig. 2(b) does not confirm this hypothesis as lines $\mathrm{A}$ and $\mathrm{C}$ are visible at earlier times than line B. A possible explanation of these data is that, as the excitation power is increased, the dot is found in different charged states leading to the observation of charged excitons alongside the neutral one.

A technique which allows to discriminate between neutral exciton and charged exciton transitions is polarization

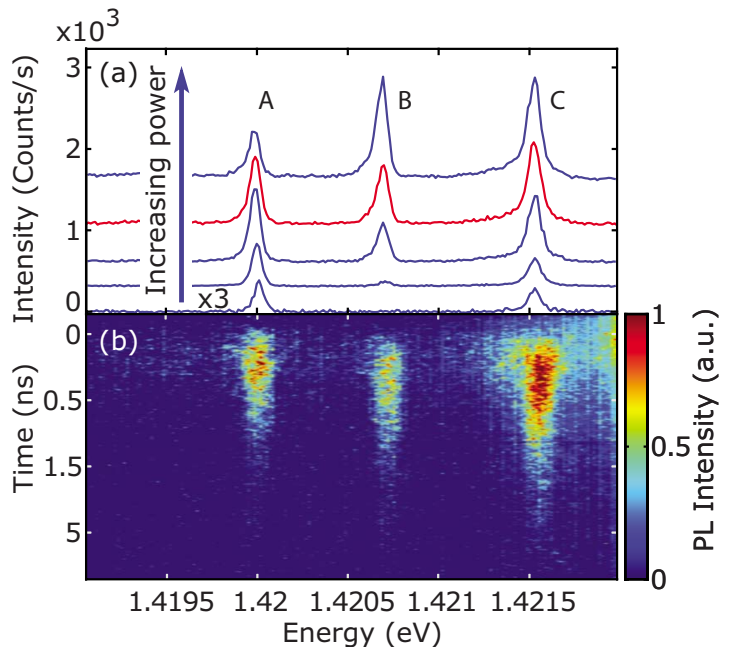

FIG. 2. (Color online) (a) PL spectra of a QD grown on a vicinal surface under pulsed laser excitation $(1.647 \mathrm{eV})$ at various powers. (b) Time resolved PL corresponding to the spectrum plotted in red.

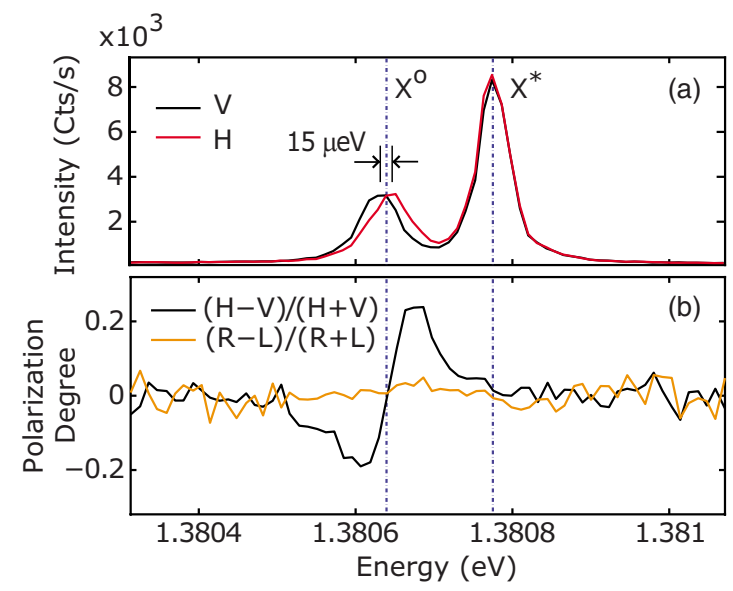

FIG. 3. (Color online) (a) PL intensity for two orthogonal linear polarizations: horizontal $(\mathrm{H})$ and vertical $(\mathrm{V})$. The two components of the low energy peak are separated in energy by $15 \mu \mathrm{eV}$ and are attributed to a neutral exciton $\left(\mathrm{X}^{\circ}\right)$. The high energy peak is not split and can therefore be attributed to a charged exciton $\left(\mathrm{X}^{*}\right)$. (b) Degree of linear (circular) polarization in black (orange).

tomography. ${ }^{22}$ By measuring the projection of the PL spectrum on six different polarization states we can reconstruct, for each energy, the polarization state of the emitted photons. The neutral exciton state is usually split in energy ${ }^{23}$ so that the corresponding $\mathrm{X}$ transitions are of slightly different energy and orthogonal linear polarizations. The charged excitons, on the contrary, are not split, due to the Kramers degeneracy, and give an unpolarized PL line. Due to this difference in polarization we can distinguish the exciton and charged exciton lines, as in Fig. 3. Here the line on the low energy side shows a polarization structure suggesting that it corresponds to the $\mathrm{X}$ transition, while the other line is unpolarized and is attributed to a charged exciton.

To summarize, we report on intense and narrow (23 $\mu \mathrm{eV}$ ) emission from single QDs grown on a vicinal substrate. Polarization measurements reveal an exciton splitting of $15 \mu \mathrm{eV}$. The good optical properties of these dots, together with the self-alignment induced by the growth technique could allow for experiments involving multiple, and possibly coupled, QDs. We show that the substrate misorientation angle can be used as an extra parameter to control the QD density and geometry, without compromising their optical properties.

This research work was partially supported by the Dutch Foundation for Fundamental Research on Matter and by the Russian Academy of Sciences (Fundamentals of Nanotechnologies and Nanomaterials Program). The authors thank Leo Kouwenhoven for support.

${ }^{1}$ T. H. Stievater, X. Li, D. G. Steel, D. Gammon, D. S. Katzer, D. Park, C. Piermarocchi, and L. J. Sham, Phys. Rev. Lett. 87, 133603 (2001).

${ }^{2}$ X. Li, Y. Wu, D. Steel, D. Gammon, T. Stievater, D. Katzer, D. Park, C. Piermarocchi, and L. Sham, Science 301, 809 (2003).

${ }^{3}$ L. Robledo, J. Elzerman, G. Jundt, M. Atature, A. Hogele, S. Falt, and A. Imamoglu, Science 320, 772 (2008).

${ }^{4}$ P. Michler, A. Imamoğlu, M. D. Mason, P. J. Carson, G. F. Strouse, and S. K. Buratto, Nature (London) 406, 968 (2000).

${ }^{5}$ Z. Yuan, B. E. Kardynal, M. R. Stevenson, A. J. Shields, C. J. Lobo, K. Cooper, N. S. Beattie, D. A. Ritchie, and M. Pepper, Science 295, 102 (2002).

${ }^{6}$ N. Akopian, N. H. Lindner, E. Poem, Y. Berlatzky, J. Avron, D. Gershoni, B. D. Gerardot, and P. M. Petroff, Phys. Rev. Lett. 96, 130501 (2006).

${ }^{7}$ R. J. Young, M. R. Stevenson, P. Atkinson, K. Cooper, D. A. Ritchie, and 
A. J. Shields, New J. Phys. 8, 29 (2006).

${ }^{8}$ R. Hafenbrak, S. M. Ulrich, P. Michler, L. Wang, A. Rastelli, and O. G. Schmidt, New J. Phys. 9, 315 (2007).

${ }^{9}$ M. Atature, J. Dreiser, A. Badolato, and A. Imamoglu, Nat. Phys. 3, 101 (2007).

${ }^{10}$ C. Santori, D. Fattal, J. Vuckovic, G. S. Solomon, and Y. Yamamoto, Nature (London) 419, 594 (2002).

${ }^{11}$ D. S. L. Mui, D. Leonard, L. A. Coldren, and P. M. Petroff, Appl. Phys. Lett. 66, 1620 (1995).

${ }^{12}$ Y. Sugiyama, Y. Nakata, K. Imamura, S. Muto, and N. Yokoyama, Jpn. J. Appl. Phys., Part 1 35, 1320 (1996).

${ }^{13}$ M. Kitamura, M. Nishioka, J. Oshinowo, and Y. Arakawa, Appl. Phys. Lett. 66, 3663 (1995).

${ }^{14}$ S. Liang, H. L. Zhu, and W. Wang, J. Appl. Phys. 100, 103503 (2006).

${ }^{15}$ S. Liang, H. Zhu, J. Pan, X. Ye, and W. Wang, J. Cryst. Growth 289, 477 (2006).

${ }^{16}$ A. A. Tonkikh, G. E. Cirlin, V. G. Dubrovskii, Yu. B. Samsonenko, N. K.
Polyakov, V. A. Egorov, A. G. Gladyshev, N. V. Kryzhanovskaya, and V. M. Ustinov, Tech. Phys. Lett. 29, 691 (2003).

${ }^{17}$ V. G. Dubrovskii, G. E. Cirlin, and V. M. Ustinov, Phys. Status Solidi B 241, R42 (2004).

${ }^{18}$ G. E. Cirlin, G. M. Guryanov, A. O. Golubok, S. Ya. Tipissev, N. N. Ledentsov, P. S. Kop'ev, M. Grundmann, and D. Bimberg, Appl. Phys. Lett. 67, 97 (1995)

${ }^{19}$ V. G. Talalaev, B. V. Novikov, G. Gobsch, R. Goldhahn, N. Stein, J. W. Tomm, A. Maassdorf, G. E. Cirlin, V. N. Petrov, and V. M. Ustinov, Phys. Status Solidi B 224, 101 (2001).

${ }^{20}$ D. V. Regelman, U. Mizrahi, D. Gershoni, E. Ehrenfreund, W. V. Shoenfeld, and P. M. Petroff, Phys. Rev. Lett. 87, 257401 (2001).

${ }^{21}$ D. L. Moehring, P. Maunz, S. Olmschenk, K. C. Younge, D. N. Matsukevich, L. M. Duan, and C. Monroe, Nature (London) 449, 68 (2007).

${ }^{22}$ J. Altepeter, D. James, and P. Kwiat, Lect. Notes Phys. 649, 113 (2004).

${ }^{23}$ D. Gammon, E. S. Snow, B. V. Shanabrook, D. S. Katzer, and D. Park, Phys. Rev. Lett. 76, 3005 (1996). 